\title{
The Relevance of Schizotypal Traits for Understanding Interpersonal Functioning in Adolescents With...
}

Article in Personality Disorders: Theory, Research, and Treatment · December 2015

DOI: $10.1037 /$ per0000163

CITATION

1

5 authors, including:

Barbara De Clercq

Ghent University

77 PUBLICATIONS 1,716 CITATIONS

SEE PROFILE

\section{Joost Hutsebaut}

De Viersprong National Institute of Personalit. .

30 PUBLICATIONS 175 CITATIONS

SEE PROFILE
READS

51

\section{Paul T van der Heijden}

Reinier van Arkel Groep

32 PUBLICATIONS 163 CITATIONS

SEE PROFILE

\section{Marcel A. G. van Aken}

Utrecht University

360 PUBLICATIONS $\quad 4,832$ CITATIONS

SEE PROFILE

Some of the authors of this publication are also working on these related projects: 


\section{Personality Disorders: Theory, Research, and Treatment}

\section{The Relevance of Schizotypal Traits for Understanding Interpersonal Functioning in Adolescents With Psychiatric Problems}

Lize Verbeke, Barbara De Clercq, Paul Van der Heijden, Joost Hutsebaut, and Marcel A. G. van Aken

Online First Publication, December 7, 2015. http://dx.doi.org/10.1037/per0000163

CITATION

Verbeke, L., De Clercq, B., Van der Heijden, P., Hutsebaut, J., \& van Aken, M. A. G. (2015, December 7). The Relevance of Schizotypal Traits for Understanding Interpersonal Functioning in Adolescents With Psychiatric Problems. Personality Disorders: Theory, Research, and

Treatment. Advance online publication. http://dx.doi.org/10.1037/per0000163 


\title{
The Relevance of Schizotypal Traits for Understanding Interpersonal Functioning in Adolescents With Psychiatric Problems
}

\author{
Lize Verbeke and Barbara De Clercq \\ Ghent University
}

\author{
Joost Hutsebaut \\ Kenniscentrum Persoonlijkheidsstoornissen De Viersprong, \\ Halsteren, the Netherlands
}

\author{
Paul Van der Heijden \\ Reinier van Arkel Mental Health Institute, 's-Hertogenbosch, the \\ Netherlands Nijmegen
}

\author{
Marcel A. G. van Aken \\ Utrecht University
}

\begin{abstract}
Social relationships are considered highly important throughout adolescence (Kenny et al., 2013), both for the further development and consolidation of identity, social roles, and skills. The schizotypal personality disorder (STPD) has a strong negative impact on these relationships with both parents and peers (Cramer et al., 2006; Hengartner et al., 2014), and can thus be considered as a risk factor for early maladaptive social functioning. The current study focuses on the relevance of different dimensional STPD traits for understanding social functioning, by examining their unique associations with global and more specific parental and peer relationship characteristics in a group of referred late-adolescents $(N=$ 205, mean age $=20.27$ ). Negative schizotypal traits, assessed by the Diagnostic and Statistical Manual of Mental Disorders (5th ed.; DSM-5; American Psychiatric Association, 2013) STPD traits Restricted Affectivity, Withdrawal, and Suspiciousness (Krueger et al., 2012) appeared to be a unique predictor for less maternal and peer social support. Positive schizotypal traits were measured with the age-specific Oddity trait scale (Verbeke \& De Clercq, 2014) and proved to be a unique predictor beyond negative schizotypal traits for negative interactions with adolescents' mother and a best friend. These results highlight the heterogeneous nature of the STPD construct and suggest that a dimensional description may contribute to a more detailed understanding of how the STPD relates to poor interpersonal relationship quality in vulnerable adolescents.
\end{abstract}

Keywords: adolescence, interpersonal functioning, Oddity, schizotypal personality disorder

Adolescence is generally considered a critical period for social development (La Greca \& Harrison, 2005), consisting of core developmental tasks that are indispensable for further psychological growth toward adulthood (DeHart, Stroufe, \& Cooper, 2004). Parents (Sheeber, Davis, Leve, Hops, \& Tildesley, 2007) and peers (Brown \& Klute, 2003; La Greca \& Harrison, 2005) are of crucial importance during this period, since relationship quality with these key figures has a large impact on adolescents' mental health (Kenny, Dooley, \& Fitzgerald, 2013). The presence of maladaptive personality characteristics may signify a major threat for the qual-

Lize Verbeke and Barbara De Clercq, Department of Developmental, Personality, and Social Psychology, Ghent University; Paul Van der Heijden, Centre for Adolescent Psychiatry, Reinier van Arkel Mental Health Institute, 's-Hertogenbosch, the Netherlands; Joost Hutsebaut, Kenniscentrum Persoonlijkheidsstoornissen De Viersprong, Halsteren, the Netherlands; Marcel A. G. van Aken, Department of Developmental Psychology, Utrecht University.

Correspondence concerning this article should be addressed to Lize Verbeke, Department of Developmental, Personality, and Social Psychology, Ghent University, Henri Dunantlaan 2, Ghent, 9000 Belgium. E-mail: lize.verbeke@ugent.be ity of these social relationships, as shown in multiple studies indicating the strong association of personality pathology with poor interpersonal functioning (Chen, Cohen, Kasen, \& Johnson, 2006; Cramer, Torgersen, \& Kringlen, 2006; Hengartner, Müller, Rodgers, Rössler, \& Ajdacic-Gross, 2014). Across all personality disorders (PDs) that are listed in the Diagnostic and Statistical Manual of Mental Disorders (5th ed.; DSM-5; American Psychiatric Association [APA], 2013), the eccentric Cluster A PDs show the strongest negative correlations with quality of contact with family and friends (Cramer et al., 2006). Within this Cluster A, the schizotypal PD (STPD) is most strongly related to problems in social interactions with different attachment figures (Hengartner et al., 2014), implicating that STPD-related pathology may signify one of the most detrimental PDs for adolescents.

Most studies have approached STPD as a unitary and categorical construct, consisting of a broad set of symptoms (APA, 2013). A compelling amount of studies, however, has already convincingly demonstrated that the heterogeneity of schizotypal symptoms is best captured in a multidimensional structure (e.g., Gross, Mellin, Silvia, Barrantes-Vidal, \& Kwapil, 2014; Kwapil, BarrantesVidal, \& Silvia, 2008; Vollema \& van den Bosch, 1995), including a "positive" and a "negative" dimension. The positive schizotypy dimension is reflecting aberrant mental activity (e.g., ideas of reference), whereas the negative symptoms comprise social anhe- 
donia and dysfunction (APA, 2013). To fully understand the significance of schizotypal pathology in interactions within the close network of adolescents, research that examines the specific and unique relevance of both components is needed. The present study focuses on this unique role of both positive and negative STPD characteristics in understanding various aspects of relational functioning with both parents and peers in a sample of late adolescents with a referred status.

\section{Cluster A Personality Pathology and Social Functioning}

Problematic social interactions are essential to all personality disorders (Hengartner et al., 2014) and are therefore explicitly mentioned as a diagnostic criterion (Criterion A) for a PD diagnosis in the current DSM-5 (APA, 2013). However, very few studies have examined the association of PDs with specific aspects of interpersonal functioning (Hengartner et al., 2014). Several studies in adults have addressed this issue more indirectly by use of the broad construct of "quality of life," because all quality of life conceptualizations systematically cover social dimensions (Hornquist, 1982; Aaronson, 1988). Other studies have approached interpersonal functioning in PDs as an aspect of "functional impairment" (Skodol et al., 2005). Across all conceptualizations of interpersonal functioning, Cluster A personality pathology appears to be one of the strongest correlates of problematic close relationships (Cramer et al., 2006, 2007; Hengartner et al., 2014). Skodol and colleagues (2005) specifically stated that patients with a Cluster A PD experience more severe impairment in social relationships than patients with borderline, avoidant or obsessivecompulsive personality disorder. More specifically, quality of life studies have demonstrated that the three Cluster A PDs are all significant negative predictors of a variety of social quality of life indicators, such as contact with family of origin and social support in case of illness (Cramer et al., 2006, 2007). Examining more specific aspects of interpersonal functioning, Hengartner and colleagues (2014) demonstrated that all three Cluster A disorders are related to living alone, distress and conflicts in friendships, and having no partner or experiencing distress in partnership (Hengartner et al., 2014).

Although Cluster A PDs share a common ground and are to some extent all associated with problematic social interactions, some notable differences have also been observed, with the most pronounced effects for STPD. For example, in the study of Hengartner et al. (2014), the STPD is the only Cluster A disorder that was negatively associated with all indicators of interpersonal functioning and was additionally predictive for not being married or having children, feeling lonely and having conflicts with a partner. These findings led the authors to conclude that schizotypal symptoms are among the most destructive traits for establishing adequate interpersonal relationships. In a related vein, also Cramer and colleagues (2006) suggested that strongest reduction of quality of life in contact with friends and family of origin was observed for the STPD.

From a developmental perspective on the relationship between STPD and interpersonal function, similar research is rather scarce, although Chen et al. (2006) demonstrated that schizotypal traits in late adolescence are the only Cluster A predictor of reduced social quality of life more than 10 years later. A few other studies confirmed that youth with STPD symptoms are experiencing a wide range of social difficulties. In a study of Mittal, Tessner, and Walker (2007), adolescents diagnosed with STPD reported significantly less social interactions with friends in real life, but engaged more in social interactions on the Internet compared with a control group of adolescents diagnosed with a different PD including other Cluster A PDs. These results may point to the fact that adolescents with STPD are socially motivated but experience an interpersonal inconvenience in real-life situations. Moreover, adolescents with schizotypal traits appear to be more at risk to be confronted with different forms of peer victimization (Fung \& Raine, 2012).

\section{Positive Versus Negative Schizotypal Traits}

Categorical PD research does not make a distinction between positive and negative STPD symptoms, but considers the STPD as a unitary construct (APA, 2013). Several studies have adopted a more dimensional approach on schizotypal traits by using the dimensional schizotypy construct (Tiliopoulos \& Goodall, 2009; Cohen \& Davis, 2009; Abbott \& Byrne, 2012) or other dimensional schizotypal trait factors (Keeley, Flanagan, \& McCluskey, 2014) that allow for a more fine-tuned description of STPD traits. The construct of "schizotypy" is widespread in the literature and is considered as a vulnerability for schizophrenia spectrum disorders, including the schizotypal personality disorder. Schizotypy is usually conceptualized as a continuum of schizophrenia spectrum-like abnormalities, ranging from nonclinical manifestations to Cluster A personality pathology, to full-blown psychosis (Kwapil et al. 2008). Empirical evidence has repeatedly pointed to a multidimensional structure of schizotypy (Gross et al., 2014), with positive and negative schizotypy as most consistently replicated factors (Kwapil et al., 2008; Kwapil, Ros-Morente, Silvia, \& BarrantesVidal, 2012). Several authors have used these dimensions of positive and negative schizotypy in order to investigate the unique influence of both symptom groups on several indices of functioning (Dinn, Harris, Aycicegi, Greene, \& Andover, 2002; Kwapil et al., 2008), including interpersonal relationships with family and friends. In this vein, Cohen and Davis (2009) demonstrated that the negative schizotypy symptoms showed the most striking unique contribution to problematic interactions with parents and peers. These results were replicated in a study of Abbott and Byrne (2012) in a sample of university students, and also in an experience sampling study with undergraduate students, negative schizotypy was characterized by social disinterest as reflected in the associations of negative schizotypal traits with greater social distance to others, feelings of less closeness and the desire to be alone (Kwapil, Brown, Silvia, Myin-Germeys, \& Barrantes-Vidal, 2012).

Beyond this consensus on the role of negative symptoms in poor interpersonal relationship quality, also positive schizotypal symptoms may contribute to difficulties in relationships with parents and peers. People high on positive schizotypy experience a social ambivalence, that can be understood from the association of positive schizotypy with an increased desire to be alone when with others but also with an increased desire to be with others when alone (Kwapil et al., 2012). Wolff (1991) stated that positive schizotypal symptoms of odd or eccentric behavior may hamper relations with peers in adolescence, and Mittal, Dhruv, Tessner, Walder, \& Walker (2007) suggest that next to negative schizotypal 
symptoms, also other STPD symptoms at a young age, such as irregular and limited gesturing (Mittal et al., 2006), motor abnormalities (Walker, Lewis, Loewy, \& Palyo, 1999; Mittal, Dhruv, et al., 2007), and problems in interpreting nonverbal cues (Logan, 1999), may complicate adequate interpersonal functioning. Recent studies confirm that both schizotypy dimensions are associated with poorer social functioning (Kwapil et al., 2008; Kwapil, Gross, Silvia, \& Barrantes-Vidal, 2013), although only negative schizotypy was predictive for social indicators that are specifically related to closeness in relationships. Also from a DSM-5 trait perspective, there is some evidence that positive-like schizotypal traits are uniquely related to communication difficulties. The higherorder Psychoticism trait, in DSM-5 Section III proposed for the assessment of positive STPD symptoms (APA, 2013), proved to be a very robust predictor of impairment in communication in a clinical sample of adults (Keeley, Flanagan, \& McCluskey, 2014).

In sum, most studies on the association of schizotypal features and interpersonal functioning highlight the relevance of negative STPD symptoms (Abbott \& Byrne, 2012; Cohen \& Davis, 2009; Henry, Bailey, \& Rendell, 2008), although preliminary evidence suggests that also positive STPD symptoms significantly contribute to problematic social interactions (Keeley et al., 2014; Mittal, Tessner, et al., 2007). The current study aims to elaborate the specific associations of different STPD traits with both parental and peer relationships in adolescence, by focusing on particular relational features, as suggested by Hengartner et al. (2014).

\section{The Current Study}

In line with several studies that have adopted the "quality of life" construct in examining the association of STPD traits and interpersonal interactions (Abbott \& Byrne, 2012; Cramer et al., 2006, 2007; Cohen \& Davis, 2009), the current study will explore in a first step the unique associations between positive versus negative STPD symptoms and parental/peer relationships. In a second step, also more specific relationship characteristics with these attachment figures will be explored, including both positive parental and peer interactions (i.e., attachment, caregiving and affiliation behavior) and negative interactions (i.e., criticism, conflicts and antagonism) with parents and peers, to obtain a more detailed understanding on the association of schizotypal symptoms with these important social interactions in adolescents' daily lives. Starting from the STPD conceptualization in the hybrid DSM-5 personality pathology (APA, 2013) model, negative STPD traits will be assessed by the proposed DSM-5 facets Restricted Affectivity, Withdrawal, and Suspiciousness, as recent evidence demonstrated the general applicability of the DSM-5 trait model in adolescents (De Clercq et al., 2014). Psychometric properties of the higher-order Psychoticism trait, however, assessing positive schizotypal characteristics (APA, 2013), behaved differently in a younger age group compared with adult findings (Krueger, Derringer, Markon, Watson, \& Skodol, 2012). This finding has led the authors to conclude that the DSM-5 Psychoticism trait factor may not be fully developmentally appropriate (De Clercq et al., 2014). Therefore, positive schizotypal characteristics will be measured by relying on the recently developed Oddity trait scale (Verbeke \& De Clercq, 2014) of the Dimensional Personality Symptom Itempool (DIPSI; De Clercq, De Fruyt, Van Leeuwen, \& Mervielde, 2006), an age-specific maladaptive trait instrument. In order to get more insight in the unique associations of STPD symptomatology with parental and peer relationship characteristics, the incremental validity of both negative and positive symptoms above and beyond each other will be explored. We expect unique incremental effects of both symptom groups in the prediction of interpersonal functioning with parents and peers.

\section{Method}

\section{Procedures and Participants}

Participants $(N=223,69.5 \%$ females $)$ were recruited from two mental health institutes in the Netherlands, which are the "Centre for Adolescent Psychiatry, Reinier Van Arkelgroep Den Bosch" and "De Viersprong, The Netherlands Institute for personality disorders." Most participants were referred by their general practitioner for assessment and treatment of psychiatric problems. All participants were asked to participate in the present study at the moment of admission. After receiving a description of the study, patients from Reinier Van Arkelgroep received an information letter with a personal login code that provided access to a protected online assessment platform. If patients from De Viersprong approved to participate, their e-mail address was passed to the research coordinator at Ghent University, who subsequently e-mailed them a unique login code to enter the online questionnaires. Approximately 750 patients were invited to participate in the present study of which 223 patients actually logged in on our assessment platform (response rate of 29.73\%).

Patients with an IQ below 85 were a priori excluded. Because the present study focused on a sample of late adolescents and young adults, we also excluded all participants younger than 16 $(n=7)$ and older than $24(n=11)$ for further analyses, resulting in a sample of 205 participants with $69.8 \%$ girls. The age of the remaining participants ranged between 16 and 24 years $(M=$ 20.27, $S D=2.21)$. Almost all participants $(n=197,96.1 \%)$ had the Dutch ethnicity, with $3.9 \%(n=8)$ of the participants reporting a foreign origin. The majority of the participants lived with one or both parents $(n=130,63.4 \%)$, whereas $17.1 \%(n=35)$ lived alone or with a partner $(n=10,4.9 \%)$. A small group $(n=15$, $7.3 \%$ ) were inpatients, residing at the mental health care institute. Finally, $7.3 \%(n=15)$ had another living situation, such as living with another family member or in a foster family. Almost half of the participants were at the moment of assessment not enrolled in an education program $(n=101,49.3 \%)$, whereas 67 participants $(32.8 \%)$ were enrolled in a secondary school program. Finally, 36 participants were following a professional $(n=27,13.2 \%)$ or academic ( $n=9,4.4 \%)$ higher-education program. Three fourths of the participants $(n=157,76.6 \%)$ filled out all measures, whereas $48(23.4 \%)$ participants had a missing value at one or more of the measures. The pattern of missing values was estimated by Little's Missing completely at random (MCAR) test, which was not significant.

\section{Measures}

Cluster A-related personality pathology. The recently released DSM-5 trait (APA, 2013) measure allows to describe negative schizotypal traits along a dimensional perspective, and proposes three facets that each describe an aspect of negative 
schizotypal symptoms (Krueger et al., 2012). Restricted Affectivity ( 7 items) is part of the higher-order Negative affect factor and describes a tendency to not experience or express emotions, whereas Withdrawal (10 items) and Suspiciousness (7 items) belong to the higher-order Detachment factor, with Withdrawal reflecting a need to keep distance from other people and Suspiciousness describing a basic mistrust of other people. Cronbach's alpha reliabilities for self-reports on these Restricted affectivity, Withdrawal, and Suspiciousness facets were acceptable, with values of $.79, .91$, and .76 , respectively.

For the assessment of positive schizotypal traits, the Oddity trait scale (Verbeke \& De Clercq, 2014) of the Dimensional Personality Symptom Itempool (DIPSI; De Clercq et al., 2006) was used, which assesses age-specific positive Cluster A-related personality disorder characteristics. This Oddity scale consists of 22 items to be answered on a 5-point Likert scale, that cluster together in four underlying facets (Oversensitivity to feelings, Extreme fantasy, Daydreaming, and Odd thoughts and behavior) and one higherorder Oddity trait factor. Oversensitivity to feelings (4 items) describes behavior that reflects an extreme openness to both inner and others' emotions, leading to overwhelming emotional experiences. Extreme fantasy ( 5 items) reflects an extreme tendency to indulge in fantasies, and to experience difficulties in differentiating between reality and fantasy. The facet Daydreaming (7 items) describes absent-minded feelings, thoughts and behavior that interfere with daily activities, whereas the facet Odd thoughts and behavior (6 items) describes thoughts and behavior that are odd, weird, or puzzling to others. Cronbach's alpha reliabilities in the current sample were acceptable, with values of .71 (Oversensitivity to feelings), .74 (Extreme Fantasy), .87 (Daydreaming), and .91 (Odd thoughts and behavior).

Quality of life-Peer and parental relations. Adolescents' general experience of the quality of their parental and peer relationships was assessed with self-reports on a quality of life measure for youth, the KIDSCREEN-27 (Kidscreen Group Europe, 2006). This instrument originally consists of 27 items on a 5-point Likert scale, with higher scores indicating a better quality of life. For the current study, we only selected the two KIDSCREEN dimensions referring to parental and peer relationships. The dimension Autonomy \& Parents consists of 7 items assessing feelings of autonomy, relations with parents and more financial issues. The Social support and Peer relations (4 items) dimension assesses positive interactions with friends. Cronbach's alpha reliabilities were adequate, with values of .78 (Autonomy \& Parents) and .83 (Social support \& Peer relations).

Parental and peer relationship characteristics. The Network of Relationships Inventory: Behavioral Systems Version (NRI-BSV; Furman \& Buhrmester, 2009) is a 24-item questionnaire that assesses eight features of close relationships. Two scales assess attachment behaviors, two scales describe caregiving behaviors and one scale assesses affiliation behaviors. Next to these positive interactions and social support scales, there are also three scales assessing negative interactions of criticism, conflict, and antagonism. Furthermore, two broadband factor scales "Social support" (mean of all positive interactions scales) and "Negative interactions" (mean of all negative interactions scales) can be calculated. Participants answered all questions three times: One time about their relationship with a mother figure, a second time about their relationship with a father figure and a last time about their rela- tionship with someone that they considered as a "best friend." Cronbach's alpha reliabilities for the maternal and paternal relationship broadband scales are excellent, with values of .92 and .93 for maternal and paternal Social support respectively, and .96 for both maternal and paternal Negative interactions. Alpha reliabilities for the peer relationship features amounted to .95 for Social support and .89 for Negative interactions.

\section{Statistical Analyses}

Bivariate correlations were calculated between negative and positive schizotypal symptoms, as well as between schizotypal characteristics, social indices of quality of life, and relationship characteristics. Because the main objective of this study was to get more insight in the unique contribution of negative and positive Cluster A-related symptoms in the prediction of interpersonal functioning, hierarchical regression analyses were conducted, with gender entered as a control variable in a first step, and the positive and negative STPD facets as a second and third block respectively. In a second regression analysis, the order of block 2 and 3 was reversed. Each of these regression analyses were separately run for the KIDSCREEN-27 (Kidscreen Group Europe, 2006) and NRIBSV (Furman \& Buhrmester, 2009) domains as dependent variables. All correlations between STPD symptoms and constructs of social functioning were corrected for the large amount of tests (i.e., Bonferroni correction).

\section{Results}

\section{Bivariate Correlations}

Table 1 demonstrates the intercorrelations between the negative and positive STPD traits. The negative STPD facet Restricted Affectivity is not significantly related to any of the Oddity facets, whereas Withdrawal demonstrates significant positive correlations with the Oddity facets Extreme Fantasy $(r=.33)$, Daydreaming $(r=.37)$ and Odd thoughts and behavior $(r=.28)$, respectively $(p<.001)$. Finally, Suspiciousness is most closely related to all Oddity facets, with correlation coefficients of .28 for Oversensitivity to feelings, .35 for Extreme Fantasy, .37 for Daydreaming, and .35 for Odd thoughts and behavior $(p<.001)$.

Bivariate correlations of the schizotypal facets with the KIDSCREEN-27 dimensions and NRI factors are reported in Table 2. Because of the large amount of tests, only correlations that were still significant after Bonferroni correction will be discussed. The negative STPD facets Withdrawal and Suspiciousness

Table 1

Bivariate Correlations Between Positive and Negative STPD facets

\begin{tabular}{lcccc}
\hline & \multicolumn{4}{c}{ Oddity facets } \\
\cline { 2 - 5 } \multicolumn{1}{c}{ PID-5 facets } & $\begin{array}{c}\text { Oversensitivity } \\
\text { to feelings }\end{array}$ & $\begin{array}{c}\text { Extreme } \\
\text { fantasy }\end{array}$ & Daydreaming & $\begin{array}{c}\text { Odd thoughts } \\
\text { and behavior }\end{array}$ \\
\hline Restricted affect & $-.16^{*}$ & .02 & $.16^{* *}$ & .14 \\
Withdrawal & .16 & $.33^{* *}$ & $.37^{* *}$ & $.28^{* *}$ \\
Suspiciousness & $.28^{* *}$ & $.35^{* *}$ & $.37^{* *}$ & $.35^{* *}$ \\
\hline
\end{tabular}

${ }^{*} p<.05 . \quad{ }^{* *} p<.01$. 
Table 2

Bivariate Correlations Between Positive and Negative STPD Traits With KIDSCREEN-27 Dimensions and NRI-BSV Factor Scores for Maternal, Paternal, and Peer Relationship

\begin{tabular}{|c|c|c|c|c|c|c|c|}
\hline \multirow[b]{3}{*}{ Scale } & \multicolumn{3}{|c|}{ Negative STPD traits } & \multicolumn{4}{|c|}{ Positive STPD traits } \\
\hline & \multicolumn{3}{|c|}{ PID-5 facets } & \multicolumn{4}{|c|}{ Oddity facets } \\
\hline & $\begin{array}{l}\text { Restricted } \\
\text { affectivity }\end{array}$ & Withdrawal & Suspiciousness & $\begin{array}{l}\text { Oversensitive } \\
\text { to feelings }\end{array}$ & $\begin{array}{l}\text { Extreme } \\
\text { fantasy }\end{array}$ & Day-dreaming & $\begin{array}{l}\text { Odd thoughts } \\
\text { and behavior }\end{array}$ \\
\hline \multicolumn{8}{|l|}{ KIDSCREEN } \\
\hline Autonomy \& parents & $-.22^{* *}$ & $-.26 * * *$ & $-.28^{* * * *}$ & $-.25^{* * * *}$ & -.16 & $-.31^{* * * *}$ & $-.23^{* *}$ \\
\hline Social support \& peers & $-.18^{*}$ & $-.39^{* * * *}$ & -.16 & .11 & .01 & .01 & .03 \\
\hline \multicolumn{8}{|l|}{ NRI mother } \\
\hline Social support & $-.33^{* * * * *}$ & -.15 & $-.16^{*}$ & -.05 & -.01 & $-.21^{*}$ & -.08 \\
\hline Negative interactions & .08 & .09 & .13 & $.16^{*}$ & .15 & $.26^{* * * * *}$ & $.26^{* * * * *}$ \\
\hline \multicolumn{8}{|l|}{ NRI father } \\
\hline Social support & $-.19^{*}$ & $-.25^{* *}$ & -.14 & -.08 & .02 & $-.20^{*}$ & -.06 \\
\hline Negative interactions & .02 & .04 & .02 & .19 & .06 & $.19^{*}$ & .07 \\
\hline \multicolumn{8}{|l|}{ NRI peer } \\
\hline Social support & -.15 & $-.31^{* * * *}$ & -.07 & $.25^{* *}$ & .16 & .12 & .08 \\
\hline Negative interactions & .00 & .15 & $.17^{*}$ & -.02 & $.24^{* * *}$ & $.20^{*}$ & $.26^{* * *}$ \\
\hline
\end{tabular}

are negatively related to Autonomy \& Parents, with correlation coefficients of -.26 and -.28 , respectively $(p<.002)$, whereas only Withdrawal demonstrates a significant negative association with Social support and peers, $r=-.39, p<.002$. The Oddity facets are generally less associated with the KIDSCREEN-27 dimensions, with only Oversensitivity to feelings and Daydreaming demonstrating a significant negative association with Autonomy \& Parents. The lower-part of Table 2 reflects the correlations between the schizotypal facets and the NRI factors for maternal, paternal, and peer relationships, and shows that high scores on Restricted Affectivity are associated with less maternal support, $r=-.33, p<.002$, whereas high scores on Daydreaming and Odd thoughts and behavior are associated with more maternal Negative interactions, $r=.26, p<.002$. In the relationship with father, none of the STPD facets is demonstrating a significant association with relationship characteristics. Finally, in the relationship with a peer, Withdrawal is negatively related to peer social support, $r=-.31, p<.002$, whereas Odd thoughts and behavior is again significantly correlated with Negative interactions, $r=.26, p<.002$. Adolescents with negative schizotypal traits, especially in terms of high Restricted Affectivity and Withdrawal, thus experience less social support from attachment figures but these similar traits are not related to more negative interactions. In contrast, positive schizotypal traits appear unrelated to social support, but are associated with more negative interactions with mother and a best friend, especially for high scores on Daydreaming and Odd thoughts and behavior.

\section{Hierarchical Regression Analyses}

Table 3 presents the results for the regressions on the KIDSCREEN-27 dimensions Autonomy \& Parents and Social support \& Peers. For both KIDSCREEN-27 dimensions, gender was not a significant predictor. The Oddity facets were entered in a second step, explaining $10 \%$ additional variance in the prediction of Autonomy \& Parents and no significant variance in the prediction of Social support and peers. The three negative STPD facets were entered in a third step and explained incremental validity above and beyond the Oddity facets for both Autonomy \& Parents (7\%) and Social support \& Peers (17\%), with Restricted Affectivity as a significant negative predictor for Autonomy \& Parents $(\beta=-.20, p<.05)$ and Withdrawal as significant negative predictor of Social support \& Peers $(\beta=-.44, p<.01)$. When reversing the sequence of step 2 and 3 , the negative STPD facets in step 2 explained for both KIDSCREEN-27 domains additional variance, amounting to $12 \%$ for Autonomy \& Parents and to $15 \%$ for Social support \& Peers. The four Oddity facets did not add any incremental variance in the prediction of both KIDSCREEN dimensions in a third step.

Tables 4 and 5 present the results of the hierarchical regressions on the NRI relationship quality domains, with Table 4 reflecting the results of the parental relationships and Table 5 including peer relationship characteristics. For the parental NRI domains, gender is only a significant predictor for maternal Negative interactions, with girls reporting higher scores than boys. When the positive STPD facets are added in step 2, they only explain a significant amount of variance for maternal Negative interactions (9\%), with Odd thoughts and behavior as a significant predictor $(\beta=.25, p<.05)$. The three negative STPD facets in step 3 explain incremental validity above and beyond gender and the positive STPD facets in the prediction of maternal Social support, with Restricted Affectivity as a significant negative predictor $(\beta=-.30, p<.01)$. Reversing the order of steps 2 and 3, the negative STPD facets predict in step 2 less social support from both mother $(11 \%)$ and father $(7 \%)$, with Restricted Affectivity as negative predictor $(\beta=-.31, p<$ $.01)$ in the prediction of maternal Social support. The four positive STPD facets in step 3 explain additional variance above gender and the negative STPD facets in the prediction of Negative interactions with the mother (7\%), with Odd thoughts and behavior as significant predictor $(\beta=.24, p<.05)$.

Regarding the relationship characteristics with a peer (see Table $5)$, gender is a significant predictor for peer Social support (10\%), 
Table 3

Multiple Hierarchical Regression Analyses KIDSCREEN-27 Dimensions, Negative STPD Facets, and Positive STPD Facets

\begin{tabular}{|c|c|c|c|c|}
\hline Criteria and predictor & $R^{2}$ & $\Delta R^{2}$ & $F_{\text {change }}$ & Predictor (standardized $\beta$ ) \\
\hline \multicolumn{5}{|l|}{ Autonomy \& parents } \\
\hline Step 1: Sex & .02 & .02 & 3.54 & $\operatorname{Sex}(-.15)$ \\
\hline \multicolumn{5}{|l|}{ Negative above positive } \\
\hline Step 2: Oddity facets & .12 & .10 & $4.34^{* *}$ & OvToFe $(-.14)$, ExFa $(.13)$, Ddr $(-.22)$, OdThBh $(-.12)$ \\
\hline Step 3: PID-5 facets & .20 & .07 & $4.53^{* *}$ & ResAff $\left(-.20^{*}\right)$, Withdr $(-.05)$, Suspi $(-.14)$ \\
\hline \multicolumn{5}{|l|}{ Positive above negative } \\
\hline Step 2: PID-5 facets & .14 & .12 & $7.22^{* *}$ & ResAff $\left(-.18^{*}\right)$, Withdr $(-.02)$, Suspi $\left(-.20^{*}\right)$ \\
\hline Step 3: Oddity facets & .20 & .05 & 2.42 & OvToFe $(-.18), \operatorname{ExFa}(.14), \operatorname{Ddr}(-.12)$, OdThBh $(-.09)$ \\
\hline \multicolumn{5}{|l|}{ Social support \& peers } \\
\hline Step 1: Sex & .01 & .01 & 1.61 & $\operatorname{Sex}(.11)$ \\
\hline \multicolumn{5}{|l|}{ Negative above positive } \\
\hline Step 2: Oddity facets & .03 & .02 & .62 & OvToFe (.15), ExFa (-.08), Ddr (-.10), OdThBh (.10) \\
\hline Step 3: PID-5 facets & .20 & .17 & $9.50^{* *}$ & ResAff $(.03)$, Withdr $\left(-.44^{* *}\right)$, Suspi $(-.05)$ \\
\hline \multicolumn{5}{|l|}{ Positive above negative } \\
\hline Step 2: PID-5 facets & .16 & .15 & $8.41^{* *}$ & ResAff $(.01)$, Withdr $\left(-.40^{* *}\right)$, Suspi $(.02)$ \\
\hline Step 3: Oddity facets & .20 & .04 & 1.47 & OvToFe (.11), ExFa (.00), Ddr (.06), OdThBh (.07) \\
\hline
\end{tabular}

Note. $\quad$ ResAff $=$ Restricted Affectivity; Withdr $=$ Withdrawal; Suspi $=$ Suspiciousness; OvToFe $=$ Oversensitivity to feelings; ExFa = Extreme Fantasy; Ddr = Daydreaming; OdThBh $=$ Odd thoughts and behavior. ${ }^{*} p<.05 . \quad{ }^{* *} p<.01$.

with girls reporting more peer support than boys. When the positive STPD facets are added in step 2, these facets explain an additional $11 \%$ of the variance in the prediction of peer Negative interactions, whereas they do not add significant variance in the prediction of peer Social support. The negative STPD facets display an incremental validity in the third step above and beyond gender and the positive STPD facets in the prediction of peer Social support $(13 \%)$, with Withdrawal as significant negative predictor $(\beta=-.41, p<.001)$. Changing the order of steps 2 and 3 demonstrates that the negative STPD facets are a significant predictor of less peer social support in step 2 (10\%, Withdrawal $\beta=-.34, p<.001)$. The positive STPD facets display an incremental validity in the third step above and beyond gender and the negative STPD facets in the prediction of peer Negative interactions (8\%), but none of the specific positive STPD facets appeared to be a significant predictor.

\section{Discussion}

The current study explored whether schizotypal traits are relevant for understanding interpersonal functioning in referred adolescents, and indicates in line with other studies a significant association with impaired relationship quality (Cramer et al., 2006; Hengartner et al., 2014). In two different ways, the current study expands previous findings in this research area. First, the significance of including different significant attachment figures has been demonstrated, as schizotypal traits behave differently in the interpersonal context, depending upon the specific relationship type that is explored (i.e., relationship with mother, father or peer). Second, the current results point to the need of specifying the kind of interaction (i.e., positive vs. negative interactions), given that the small but unique associations of positive and negative schizotypal traits with impaired social functioning flow from different components, with the negative schizotypal traits mainly relating to less positive interactions and the positive schizotypal traits con- tributing to a higher rate of negative interactions across attachment figures.

\section{Schizotypal Traits and Their Associations Across Attachment Figures}

The current study confirms previous findings that have pointed to the negative association of adolescent schizotypal traits and parental relationship quality. Moreover, our results underscore the relevance of examining maternal and paternal relationship characteristics separately, because schizotypal traits appear to be only related to the adolescent-mother relationship and are unrelated to paternal relationship characteristics. This finding can possibly be framed within normative developmental attachment pathways that implicate a less secure and more distant attachment style with the father during adolescence (Doyle, Lawford, \& Markiewicz, 2009), and would not have been noticed when traditional quality of life measures that subsume parental characteristics in one variable were used. The relevance of a detailed relationship assessment can also be demonstrated at the level of peer relationships, with negative schizotypal traits as significant negative correlates of overall peer relationship quality, whereas also positive schizotypal traits pop up as a correlate when exclusively focusing on the relationship with a best friend. Adolescents with high positive schizotypal traits hence experience more negative interactions with their best friend, but these same schizotypal traits are not related to social dysfunction in the broader context of peer-functioning.

The significance of each of the more specific STPD facets also showed to be different across attachment figures. Across measures, Restricted Affectivity appears to be the unique correlate of impaired parental relationship quality, whereas Withdrawal is the relevant facet for understanding reduced peer relationship quality. Because establishing peer relationships requires a proactive engagement of an adolescent, it is not surprising that withdrawn behavior is mainly related to impaired quality of peer relations. In 
Table 4

Multiple Hierarchical Regression Analyses NRI Factors for Parental Relationships, Negative STPD Facets, and Positive STPD Facets

\begin{tabular}{|c|c|c|c|c|}
\hline Criteria and predictor & $R^{2}$ & $\Delta R^{2}$ & $F_{\text {change }}$ & Predictor (standardized $\beta$ ) \\
\hline \multicolumn{5}{|l|}{ Social support mother } \\
\hline Step 1: Sex & .01 & .01 & .90 & $\operatorname{Sex}(.08)$ \\
\hline \multicolumn{5}{|l|}{ Negative above positive } \\
\hline Step 2: Oddity facets & .07 & .06 & 2.36 & OvToFe (.01), ExFa (.16), Ddr $\left(-.32^{* *}\right)$, OdThBh (.04) \\
\hline Step 3: PID-5 facets & .15 & .08 & $4.59^{*}$ & ResAff $\left(-.30^{* * *}\right)$, Withdr $(.07)$, Suspi $(-.10)$ \\
\hline \multicolumn{5}{|l|}{ Positive above negative } \\
\hline Step 2: PID-5 facets & .11 & .11 & $5.62^{* *}$ & ResAff $\left(-.31^{* *}\right)$, Withdr $(.04)$, Suspi $(-.11)$ \\
\hline Step 3: Oddity facets & .15 & .04 & 1.69 & OvToFe $(-.07)$, ExFa $(.15), \mathrm{Ddr}(-.24 *)$, OdThBh $(.08)$ \\
\hline \multicolumn{5}{|c|}{ Negative interactions mother } \\
\hline Step 1: Sex & .04 & .04 & $5.38^{*}$ & $\operatorname{Sex}\left(.19^{*}\right)$ \\
\hline \multicolumn{5}{|l|}{ Negative above positive } \\
\hline Step 2: Oddity facets & .12 & .09 & $3.58^{*}$ & OvToFe (.00), ExFa (-.13), Ddr (.16), OdThBh $\left(.25^{*}\right)$ \\
\hline Step 3: PID-5 facets & .13 & .00 & .19 & ResAff $(.07)$, Withdr $(-.04)$, Suspi $(.02)$ \\
\hline \multicolumn{5}{|l|}{ Positive above negative } \\
\hline Step 2: PID-5 facets & .05 & .02 & .94 & ResAff (.08), Withdr (-.00), Suspi (.09) \\
\hline Step 3: Oddity facets & .13 & .07 & $2.91^{*}$ & OvToFe (.02), ExFa (-.12), Ddr (.15), OdThBh $\left(.24^{*}\right)$ \\
\hline \multicolumn{5}{|l|}{ Social support father } \\
\hline Step 1: Sex & .00 & .00 & .17 & $\operatorname{Sex}(-.03)$ \\
\hline \multicolumn{5}{|l|}{ Negative above positive } \\
\hline Step 2: Oddity facets & .06 & .06 & 2.38 & OvToFe $(-.02), \operatorname{ExFa}(.20), \operatorname{Ddr}\left(-.31^{* *}\right)$, OdThBh $(.02)$ \\
\hline Step 3: PID-5 facets & .11 & .05 & 2.53 & ResAff $(.07)$, Withdr $(-.04)$, Suspi $(.02)$ \\
\hline \multicolumn{5}{|l|}{ Positive above negative } \\
\hline Step 2: PID-5 facets & .07 & .07 & $3.49^{*}$ & ResAff $(-.12)$, Withdr $(-.18)$, Suspi (.02) \\
\hline Step 3: Oddity facets & .11 & .04 & 1.68 & OvToFe $(-.06), \operatorname{ExFa}(.22), \operatorname{Ddr}(-.23)$, OdThBh $(.03)$ \\
\hline \multicolumn{5}{|l|}{ Negative interactions father } \\
\hline Step 1: Sex & .02 & .02 & 2.75 & $\operatorname{Sex}(.14)$ \\
\hline \multicolumn{5}{|l|}{ Negative above positive } \\
\hline Step 2: Oddity facets & .05 & .03 & 1.30 & OvToFe (.03), ExFa (-.07), Ddr (.23), OdThBh $(-.04)$ \\
\hline Step 3: PID-5 facets & .06 & .00 & 1.00 & ResAff $(.05)$, Withdr $(-.01)$, Suspi $(-.03)$ \\
\hline \multicolumn{5}{|l|}{ Positive above negative } \\
\hline Step 2: PID-5 facets & .02 & .01 & .22 & ResAff (.05), Withdr (.02), Suspi (.00) \\
\hline Step 3: Oddity facets & .06 & .03 & 1.18 & OvToFe $(.04), \operatorname{ExFa}(-.06), \operatorname{Ddr}(.22)$, OdThBh $(-.04)$ \\
\hline
\end{tabular}

contrast, an adolescent cannot entirely withdraw from the relationship with his or her mother. The mother-child relationship is by definition rather unconditional and is generally considered as an intimate understanding with room for expressing emotionally related issues. The association between (experienced) less social support and the lack of affective expression can be framed from this perspective, with mutual influences between both actors that are involved. It may be the case that, because of the child's restricted affective expression, mothers are no longer able to adequately respond to the need for social support of their child. Vice versa, attachment theory research has demonstrated that children with less responsive and supportive mothers become less emotionally competent (Colle \& Del Giudice, 2011), indicating that adolescents' restricted emotional pattern in the mother-child relationship rather represents a learning effect of a nonresponsive maternal parenting style. Since all dyadic relationships are characterized by mutual influences (Kashy \& Kenny, 2000), with interaction partners affecting each other's cognitions, emotions, and behaviors, both mother and child probably contribute to this negative interactive process, putting youngsters with negative schizotypal traits at risk for missing the highly important maternal social support at that age.

\section{Schizotypal Traits and Their Associations With Different Kinds of Interactions}

Schizotypal traits and relationship characteristics were dimensionally assessed and grouped in conceptually different subcomponents, that is, positive versus negative schizotypal traits and positive versus negative interactions, with a clear correlation pattern across attachment figures. First, negative schizotypal traits proved to be consistently associated with less experienced positive interactions with the mother and with a best friend. These results are in line with previous studies that have indicated the significant effect of negative schizotypy features in the prediction of less satisfaction with personal relationships (Abbott \& Byrne, 2012; Cohen \& Davis, 2009). Second, positive STPD traits were consistently related to higher rates of negative interactions, with Odd thoughts and behavior and Oversensitivity to feelings as significant predictors in the relationship with the mother and a best friend respectively.

These findings may imply that the distress and conflicts in social interactions as often reported in the STPD (Hengartner et al., 2014; Cramer et al., 2006) are in particular connected to the positive schizotypal symptoms. Evidence for this hypothesis can be found 
Table 5

Multiple Hierarchical Regression Analyses NRI Factors for Peer Relationships, Negative STPD Facets, and Positive STPD Facets

\begin{tabular}{|c|c|c|c|c|}
\hline Criteria and predictor & $R^{2}$ & $\Delta R^{2}$ & $F_{\text {change }}$ & Predictor (standardized $\beta$ ) \\
\hline \multicolumn{5}{|l|}{ Social support peer } \\
\hline Step 1: Sex & .10 & .10 & $15.16^{* *}$ & $\operatorname{Sex}\left(.31^{* *}\right)$ \\
\hline \multicolumn{5}{|l|}{ Negative above positive } \\
\hline Step 2: Oddity facets & .14 & .04 & 1.57 & OvToFe (.16), ExFa (.11), Ddr (-.02), OdThBh $(-.04)$ \\
\hline Step 3: PID-5 facets & .27 & .13 & $7.90^{* * *}$ & ResAff $(-.07)$, Withdr $\left(-.41^{* *}\right)$, Suspi $(-.02)$ \\
\hline \multicolumn{5}{|l|}{ Positive above negative } \\
\hline Step 2: PID-5 facets & .19 & .10 & $5.39^{* * *}$ & ResAff $(-.02)$, Withdr $\left(-.34^{* *}\right)$, Suspi $(.07)$ \\
\hline Step 3: Oddity facets & .27 & .08 & $3.41^{*}$ & OvToFe (.12), ExFa (.16), Ddr (.12), OdThBh $(-.04)$ \\
\hline \multicolumn{5}{|l|}{ Negative interactions peer } \\
\hline Step 1: Sex & .00 & .00 & .11 & $\operatorname{Sex}(.01)$ \\
\hline \multicolumn{5}{|l|}{ Negative above positive } \\
\hline Step 2: Oddity facets & .11 & .11 & $4.09^{* * *}$ & OvToFe $\left(-.23^{*}\right), \mathrm{ExFa}(.18), \mathrm{Ddr}(.13), \mathrm{OdThBh}(.15)$ \\
\hline Step 3: PID-5 facets & .13 & .02 & 1.16 & ResAff $(-.11)$, Withdr (.03), Suspi $(.15)$ \\
\hline \multicolumn{5}{|l|}{ Positive above negative } \\
\hline Step 2: PID-5 facets & .05 & .05 & 2.25 & ResAff $(-.08)$, Withdr (.09), Suspi (.18) \\
\hline Step 3: Oddity facets & .13 & .08 & $3.18^{*}$ & OvToFe $\left(-.27^{*}\right), \operatorname{ExFa}(.15), \operatorname{Ddr}(.13)$, OdThBh (.13) \\
\hline
\end{tabular}

Note. $\quad$ ResAff $=$ Restricted Affectivity; Withdr $=$ Withdrawal; Suspi $=$ Suspiciousness; OvToFe $=$ Oversensitivity to feelings; $\mathrm{ExFa}=$ Extreme Fantasy; Ddr $=$ Daydreaming; OdThBh $=$ Odd thoughts and behavior. ${ }^{*} p<.05 .{ }^{* * *} p<.01$.

in research demonstrating that positive schizotypal symptoms frequently co-occur with aggressive behavior (Raine, Fung, \& Lam, 2011), and are associated with high impulsivity scores (Dinn et al., 2002) and paranoid traits (Barrantes-Vidal, Chun, Myin-Germeys, \& Kwapil, 2013; Kwapil et al., 2012), that may drive the high level of conflicts. An explanation for this association has been searched in the underlying cognitive processes that are important for successful social interactions, such as emotion recognition (Moskowitz, 2005). Recent research indicates in this regard that especially positive schizotypal symptoms are associated with difficulties in "reading" the emotions of others in everyday social interactions, because of perceptual aberrations (Abbott \& Byrne, 2013). A cognitive mechanism that flows from these positive STPD symptoms and systematically gives everyday social cues a more negative or hostile connotation may hence be partly responsible for the reported adversarial and aggressive interactions with the mother and a best friend, at least in the adolescents' experience.

\section{Schizotypal Trait Assessment}

Although most research on the multidimensional nature of schizotypal personality traits has used established measures of the schizotypy construct, including the Wisconsin Schizotypy Scales (WSS; Chapman et al., 1976, 1978; Eckblad \& Chapman, 1983) and the Schizotypal Personality Questionnaire (SPQ; Raine, 1991), the current study has adopted an alternative assessment approach. The assessment of negative schizotypal traits was based on the proposal included in the DSM-5 section 3 (APA, 2013), comprising the three facets Restricted Affectivity, Withdrawal, and Suspiciousness. The allocation of Suspiciousness in the "negative" trait dimension is questionable though, because research with other schizotypy measures has demonstrated that Suspiciousness is also part of the cognitive-perceptual positive schizotypy factor (Gross et al., 2014). Our results confirm this less unidimensional nature of Suspiciousness, since this negative schizotypal trait demonstrates the highest intercorrelations with positive schizotypal facets.
Because the DSM-5 proposal for the assessment of positive schizotypal traits appeared to be developmentally inappropriate (De Clercq et al., 2014), the age-specific Oddity scale (Verbeke \& De Clercq, 2014) of the Dimensional Personality Symptom Itempool (DIPSI; De Clercq et al., 2006) was used. Although this scale is considered promising for the assessment of positive schizotypal characteristics in youth (Verbeke \& De Clercq, 2014), it should be mentioned that this conceptualization of positive schizotypal traits differs from those of the above-described traditional schizotypy measures. More specifically, the Oddity facets Oversensitivity to feelings and Daydreaming are not presented in other positive schizotypy measures, and may represent developmentally expressions of positive schizotypy traits in adulthood. Moreover, one of the positive schizotypy facets (Odd thoughts and behavior) of the age-specific Oddity scale also conceptually aligns with a third dimension of disorganized behavior that is often used in schizotypy research, which subsumes odd and eccentric behavior and odd speech (Raine, 1991). Previous research has pointed to the significance of these disorganized schizotypal traits for understanding diminished relationship quality (Abbott \& Byrne, 2012; Cohen \& Davis, 2009). Although the currently used scale structures the facet of Odd thoughts and behavior differently compared to adult schizotypy taxonomies, the results confirm its relevance in describing interpersonal conflicts as they help to explain negative interactions with mother.

\section{Limitations and Directions for Future Research}

Several limitations have to be taken into account when interpreting the current data. First, only the perspective of the adolescent was assessed, resulting in an exclusive assessment of the adolescents' subjective experiences that may be biased by their psychopathological status. Second, the present findings are crosssectional and do not provide causal evidence on the influence of schizotypal traits on parental and peer relationships. Third, we did not include relationship characteristics with a romantic partner that 
may also play an important role at this age (Shulman \& Scharf, 2000), and would have provided a more inclusive picture on adolescent STPD traits and relational functioning. Future studies may address this issue from a longitudinal perspective, as it can be hypothesized that early STPD symptoms may have a negative impact on this adolescent romantic relationship quality, and form the roots of negative relational functioning in adult couples, as recently described in a sample of married couples (South, 2014). Forth, the current study did not take into account co-occurring psychopathology such as depression that may also influence peer and parental relationship quality (Enfoux et al., 2013). Finally, it may be interesting to further elaborate the adolescent-father relationship in adolescents with vulnerable trait profiles and to explore the potential interaction effect between negative and positive schizotypal traits. In this regard, recent work has pointed to crucial interactions between schizotypy dimensions during adolescent development (Debbané \& Barrantes-Vidal, 2015). Although we did not investigate these interaction effects because of the limited sample size in the current study, this may be a highly promising avenue for future work.

\section{Conclusion}

The results of the current study suggest that STPD traits may play a role in the dyadic interactions between late adolescents and their mothers and friends. By assessing specific aspects of parental and peer relationship characteristics, we were able to delineate more detailed associations of both negative and positive STPD traits with experienced relationship quality across different attachment figures. Whereas the social-inhibited STPD traits are associated with the experience of less positive interactions, odd and eccentric traits appear to be particularly relevant for the understanding of negative social interactions within the close network of a group of vulnerable adolescents. These results also underscore the relevance of a comprehensive maladaptive trait assessment in terms of STPD traits, because different STPD subcomponents appear to independently contribute to social maladjustment.

\section{References}

Aaronson, N. K. (1988). Quantitative issues in health-related quality of life assessment. Health Policy, 10, 217-230. http://dx.doi.org/10.1016/01688510(88)90058-9

Abbott, G. R., \& Byrne, L. K. (2012). Schizotypy and subjective wellbeing in university students. Psychiatry Research, 196, 154-156. http:// dx.doi.org/10.1016/j.psychres.2011.08.013

Abbott, G., \& Byrne, L. K. (2013). Schizotypal traits are associated with poorer identification of emotions from dynamic stimuli. Psychiatry Research, 207, 40-44. http://dx.doi.org/10.1016/j.psychres.2013.03.005

American Psychiatric Association. (2013). Diagnostic and statistical manual of mental disorders (5th ed.). Arlington, VA: Author.

Barrantes-Vidal, N., Chun, C. A., Myin-Germeys, I., \& Kwapil, T. R. (2013). Psychometric schizotypy predicts psychotic-like, paranoid, and negative symptoms in daily life. Journal of Abnormal Psychology, 122, 1077-1087. http://dx.doi.org/10.1037/a0034793

Brown, B. B., \& Klute, C. (2003). Friendships, cliques and crowds. In G. R. Adams \& M. D. Berzonsky (Eds.), Blackwell handbook of adolescence (pp. 330-348). Oxford, UK: Blackwell.

Chapman, L. J., Chapman, J. P., \& Raulin, M. L. (1976). Scales for physical and social anhedonia. Journal of Abnormal Psychology, 85, 374-382.
Chapman, L. J., Chapman, J. P., \& Raulin, M. L. (1978). Body-image aberration in Schizophrenia. Journal of Abnormal Psychology, 87, 399407. http://dx.doi.org/10.1037/0021-843X.87.4.399

Chen, H., Cohen, P., Kasen, S., \& Johnson, J. G. (2006). Adolescent axis I and personality disorders predict quality of life during young adulthood. Journal of Adolescent Health, 39, 14-19. http://dx.doi.org/10 $.1016 /$ j.jadohealth.2005.07.005

Cohen, A. S., \& Davis, T. E., III. (2009). Quality of life across the schizotypy spectrum: Findings from a large nonclinical adult sample. Comprehensive Psychiatry, 50, 408-414. http://dx.doi.org/10.1016/j .comppsych.2008.11.002

Colle, L., \& Del Giudice, M. (2011). Patterns of attachment and emotional competence in middle childhood. Social Development, 20, 51-72. http:// dx.doi.org/10.1111/j.1467-9507.2010.00576.x

Cramer, V., Torgersen, S., \& Kringlen, E. (2006). Personality disorders and quality of life. A population study. Comprehensive Psychiatry, 47, 178-184. http://dx.doi.org/10.1016/j.comppsych.2005.06.002

Cramer, V., Torgersen, S., \& Kringlen, E. (2007). Socio-demographic conditions, subjective somatic health, Axis I disorders and personality disorders in the common population: The relationship to quality of life. Journal of Personality Disorders, 21, 552-567. http://dx.doi.org/10 .1521/pedi.2007.21.5.552

Debbané, M., \& Barrantes-Vidal, N. (2015). Schizotypy from a developmental perspective. Schizophrenia Bulletin, 41, S386-S395. http://dx doi.org/10.1093/schbul/sbu175

De Clercq, B., De Fruyt, F., De Bolle, M., Van Hiel, A., Markon, K. E., \& Krueger, R. F. (2014). The hierarchical structure and construct validity of the PID-5 trait measure in adolescence. Journal of Personality, 82, 158-169. http://dx.doi.org/10.1111/jopy.12042

De Clercq, B., De Fruyt, F., Van Leeuwen, K., \& Mervielde, I. (2006). The structure of maladaptive personality traits in childhood: A step toward an integrative developmental perspective for DSM-V. Journal of Abnormal Psychology, 115, 639-657. http://dx.doi.org/10.1037/0021-843X.115.4 .639

DeHart, G. B., Stroufe, A., \& Cooper, R. G. (2004). Child development: Its nature and course. New York, NY: McGraw-Hill.

Dinn, W. M., Harris, C. L., Aycicegi, A., Greene, P., \& Andover, M. S. (2002). Positive and negative schizotypy in a student sample: Neurocognitive and clinical correlates. Schizophrenia Research, 56, 171-185. http://dx.doi.org/10.1016/S0920-9964(01)00230-4

Doyle, A. B., Lawford, H., \& Markiewicz, D. (2009). Attachment style with mother, father, best friend and romantic partner during adolescence. Journal of Research on Adolescence, 19, 690-714. http://dx.doi.org/10 $.1111 / \mathrm{j} .1532-7795.2009 .00617 . \mathrm{x}$

Eckblad, M., \& Chapman, L. J. (1983). Magical ideation as an indicator of schizotypy. Journal of Consulting and Clinical Psychology, 51, 215225. http://dx.doi.org/10.1037/0022-006X.51.2.215

Enfoux, A., Courtois, R., Duijsens, I., Reveillere, C., Senon, J. L., Magnin, G., . . El-Hage, W. (2013). Comorbidity between personality disorders and depressive symptomatology in women: A cross-sectional study of three different transitional life stages. Personality and Mental Health, 7 , 233-241. http://dx.doi.org/10.1002/pmh.1228

Fung, A. L. C., \& Raine, A. (2012). Peer victimization as a risk factor for schizotypal personality in childhood and adolescence. Journal of Personality Disorders, 26, 428-434. http://dx.doi.org/10.1521/pedi.2012 .26 .3 .428

Furman, W., \& Buhrmester, D. (2009). The network of relationships inventory: Behavioral systems version. International Journal of Behavioral Development, 33, 470-478. http://dx.doi.org/10.1177/ 0165025409342634

Gross, G. M., Mellin, J., Silvia, P. J., Barrantes-Vidal, N., \& Kwapil, T. R. (2014). Comparing the factor structure of the Wisconsin schizotypy scales and the schizotypal personality questionnaire. Personality Disor- 
ders: Theory, Research, and Treatment, 5, 397-405. http://dx.doi.org/ 10.1037/per0000090

Hengartner, M. P., Müller, M., Rodgers, S., Rössler, W., \& Ajdacic-Gross, V. (2014). Interpersonal functioning deficits in association with $D S M-I V$ personality disorder dimensions. Social Psychiatry and Psychiatric Epidemiology, 49, 317-325. http://dx.doi.org/10.1007/s00127-013-0707-x

Henry, J. D., Bailey, P. E., \& Rendell, P. G. (2008). Empathy, social functioning and schizotypy. Psychiatry Research, 160, 15-22. http://dx .doi.org/10.1016/j.psychres.2007.04.014

Hörnquist, J. O. (1982). The concept of quality of life. Scandinavian Journal of Social Medicine, 10, 57-61.

Kashy, D. A., \& Kenny, D. A. (2000). The analysis of data from dyads and groups. In H. T. Reis \& C. M. Judd (Eds.), Handbook of research methods in social psychology (pp. 451-477). New York, NY: Cambridge University Press.

Keeley, J. W., Flanagan, E. H., \& McCluskey, D. L. (2014). Functional impairment and the $D S M-5$ dimensional system for personality disorder. Journal of Personality Disorders, 28, 657-674. http://dx.doi.org/10 .1521/pedi_2014_28_133

Kenny, R., Dooley, B., \& Fitzgerald, A. (2013). Interpersonal relationships and emotional distress in adolescence. Journal of Adolescence, 36, 351-360. http://dx.doi.org/10.1016/j.adolescence.2012.12.005

Kidscreen Group Europe. (2006). The Kidscreen questionnaires. Quality of life questionnaires for children and adolescents-handbook. Lengerich, Germany: Pabst Science Publisher.

Krueger, R. F., Derringer, J., Markon, K. E., Watson, D., \& Skodol, A. E. (2012). Initial construction of a maladaptive personality trait model and inventory for. DSM-5. Psychological Medicine, 42, 1879-1890. http:// dx.doi.org/10.1017/S0033291711002674

Kwapil, T. R., Barrantes-Vidal, N., \& Silvia, P. J. (2008). The dimensional structure of the Wisconsin Schizotypy Scales: Factor identification and construct validity. Schizophrenia Bulletin, 34, 444-457. http://dx.doi .org/10.1093/schbul/sbm098

Kwapil, T. R., Brown, L. H., Silvia, P. J., Myin-Germeys, I., \& BarrantesVidal, N. (2012). The expression of positive and negative schizotypy in daily life: An experience sampling study. Psychological Medicine, 42, 2555-2566. http://dx.doi.org/10.1017/S0033291712000827

Kwapil, T. R., Gross, G. M., Silvia, P. J., \& Barrantes-Vidal, N. (2013). Prediction of psychopathology and functional impairment by positive and negative schizotypy in the Chapmans' ten-year longitudinal study. Journal of Abnormal Psychology, 122, 807-815. http://dx.doi.org/10 $.1037 / \mathrm{a} 0033759$

Kwapil, T. R., Ros-Morente, A., Silvia, P. J., \& Barrantes-Vidal, N. (2012). Factor invariance of psychometric schizotypy in Spanish and American samples. Journal of Psychopathology and Behavioral Assessment, 34, 145-152. http://dx.doi.org/10.1007/s10862-011-9258-1

La Greca, A. M., \& Harrison, H. M. (2005). Adolescent peer relations, friendships, and romantic relationships: Do they predict social anxiety and depression? Journal of Clinical Child and Adolescent Psychology, 34, 49-61. http://dx.doi.org/10.1207/s15374424jccp3401_5

Logan, C. B. (1999). Emotion recognition and schizotypal personality disorder. Atlanta, GA: Emory University.

Mittal, V. A., Dhruv, S., Tessner, K. D., Walder, D. J., \& Walker, E. F. (2007). The relations among putative biorisk markers in schizotypal adolescents: Minor physical anomalies, movement abnormalities, and salivary cortisol. Biological Psychiatry, 61, 1179-1186. http://dx.doi .org/10.1016/j.biopsych.2006.08.043

Mittal, V. A., Tessner, K. D., McMillan, A. L., Delawalla, Z., Trottman, H., \& Walker, E. (2006). Gesture behavior in unmedicated schizotypal adolescents. Journal of Abnormal Psychology, 115, 351-358. http://dx .doi.org/10.1037/0021-843X.115.2.351

Mittal, V. A., Tessner, K. D., \& Walker, E. F. (2007). Elevated social Internet use and schizotypal personality disorder in adolescents. Schizophrenia Research, 94, 50-57. http://dx.doi.org/10.1016/j.schres.2007.04 .009

Moskowitz, G. B. (2005). Social cognition: Understanding self and others. New York, NY: Guilford Press.

Raine, A. (1991). The SPQ: A scale for the assessment of schizotypal personality based on DSM-III-R criteria. Schizophrenia Bulletin, 17, 555-564. http://dx.doi.org/10.1093/schbul/17.4.555

Raine, A., Fung, A. L. C., \& Lam, B. Y. H. (2011). Peer victimization partially mediates the schizotypy-aggression relationship in children and adolescents. Schizophrenia Bulletin, 37, 937-945. http://dx.doi.org/10 $.1093 / \mathrm{schbul} / \mathrm{sbr} 082$

Sheeber, L. B., Davis, B., Leve, C., Hops, H., \& Tildesley, E. (2007). Adolescents' relationships with their mothers and fathers: Associations with depressive disorder and subdiagnostic symptomatology. Journal of Abnormal Psychology, 116, 144-154. http://dx.doi.org/10.1037/0021843X.116.1.144

Shulman, S., \& Scharf, M. (2000). Adolescent romantic behaviours and perceptions: Age- and gender-related differences, and links with family and peer relationships. Journal of Research on Adolescence, 10, 99-118. http://dx.doi.org/10.1207/SJRA1001_5

Skodol, A. E., Pagano, M. E., Bender, D. S., Shea, M. T., Gunderson, J. G., Yen, S., . . McGlashan, T. H. (2005). Stability of functional impairment in patients with schizotypal, borderline, avoidant, or obsessivecompulsive personality disorder over two years. Psychological Medicine, 35, 443-451. http://dx.doi.org/10.1017/S003329170400354X

South, S. C. (2014). Personality pathology and daily aspects of marital functioning. Personality Disorders: Theory, Research, and Treatment, 5, 195-203. http://dx.doi.org/10.1037/per0000039

Tiliopoulos, N., \& Goodall, K. (2009). The neglected link between adult attachment and schizotypal personality traits. Personality and Individual Differences, 47, 299-304. http://dx.doi.org/10.1016/j.paid.2009.03.017

Verbeke, L., \& De Clercq, B. (2014). Integrating oddity traits in a dimensional model for personality pathology precursors. Journal of Abnormal Psychology, 123, 598-612. http://dx.doi.org/10.1037/a0037166

Vollema, M. G., \& van den Bosch, R. J. (1995). The multidimensionality of schizotypy. Schizophrenia Bulletin, 21, 19-31. http://dx.doi.org/10 $.1093 / \mathrm{schbul} / 21.1 .19$

Walker, E., Lewis, N., Loewy, R., \& Palyo, S. (1999). Motor dysfunction and risk for schizophrenia. Development and Psychopathology, 11, 509-523. http://dx.doi.org/10.1017/S0954579499002187

Wolff, S. (1991). 'Schizoid' personality in childhood and adult life. I: The vagaries of diagnostic labelling. The British Journal of Psychiatry, 159, 615-620, 634-635. http://dx.doi.org/10.1192/bjp.159.5.615 